\title{
Tratamiento de los tumores cerebrales intrínsecos de áreas motoras elocuentes. Resultados de un protocolo basado en la navegación, tractografía y monitorización neurofisiológica de estructuras corticales y subcorticales
}

J.M. González-Darder; P. González-López; F. Talamantes-Escribá; G. García-March; P. Roldán-Badía; V. Quilis-Quesada; F. Verdú-López; V. Bordes-García; L. Botella-Maciá; G. Masbout; V. Cortés-Doñate* y V. Belloch-Ugarte**

Servicios de Neurocirugía y Neurofisiología*. Hospital Clínico Universitario. Valencia. ERESA**. Valencia.

Resumen

Objetivos. El papel actual del tratamiento microquirúrgico de los tumores cerebrales intrínsecos se basa en alcanzar la máxima resección volumétrica del tumor minimizando la morbilidad postoperatoria. El propósito del trabajo es estudiar los beneficios de un protocolo diseñado para tratar tumores localizados en áreas elocuentes motoras, en el que se incluye la navegación y la estimulación de tractos motores subcorticales.

Material y métodos. Se han incluido 17 pacientes con tumores corticales y subcorticales de área motora tratados quirúrgicamente. Para la planificación preoperatoria se fusionaron en el sistema de navegación estudios anatómicos, de resonancia funcional motora (RNM-f) y los tractos subcorticales generados por estudios de tensor de difusión (DTI). La monitorización intraoperatoria incluía el mapeo motor por estimulación cortical y subcortical directa (ECD y EsCD) e identificación del surco central por inversión de la onda N20 con electrodos corticales multipolares. La localización de los puntos con respuesta positiva a la ECD o EsCD se correlacionaba con las áreas corticales o tractos funcionales motores definidos en los estudios preoperatorios gracias al navegador.

Resultados. La resección volumétrica tumoral media fue del $89.1 \pm 14.2 \%$ del volumen tumoral calculado en los estudios preoperatorios, con resección total $(\geq 100 \%)$ en doce pacientes. En el preoperatorio había focalidad neurológica deficitaria motora en el $\mathbf{5 8 . 8 \%}$ de los pacientes, que aumentó al $76.5 \%$ a las 24 horas de la cirugía y se redujo a los 30 días al $41.1 \%$. Hubo una gran correlación entre los datos anatómicos y funcionales, tanto a nivel cortical como subcortical. Sin embargo, en seis casos no se pudo identificar anatómicamente el surco central y en muchos pacientes la RNM-f ofrecía datos contradictorios. Se realizaron un total de 52 ECD con respuesta motora positiva que identificaba el área motora primaria, alcanzándose una correlación posi-

Recibido: 27-07-10. Aceptado: 26-09-10 tiva del $83.7 \%$. Se realizaron un total de 55 EsCD con respuesta motora positiva que identificaban tractos corticoespinales procedentes del área motora primaria. La distancia media entre los puntos de respuesta y la ubicación de los haces en el navegador era de $7.3 \pm 3.1 \mathrm{~mm}$.

Conclusiones. La integración de estudios anatómi$\cos \mathbf{y}$ funcionales preoperatorios e intraoperatorios permite una resección funcional que amplía de forma significativa la resección tumoral de los tumores alojados en áreas elocuentes motoras. La navegación permite integrar y reconocer la correlación entre los datos preoperatorios y los hallazgos intraoperatorios. Las áreas funcionales motoras corticales se reconocen anatómica y funcionalmente en el preoperatorio mediante estudios de RNM y RNM-f y las subcorticales con TDI y la generación de la tractografía a partir del mismo, mientras que la confirmación intraoperatoria se consigue mediante la ECD y estudio de inversión de la onda N20 para las áreas corticales y con la EsCD para las subcorticales. El tratamiento microquirúrgico guiado por navegación y con la ayuda de los estudios descritos permite resecciones tumorales medias del $\mathbf{9 0 \%}$ en lesiones tumorales de áreas motoras corticales y subcorticales elocuentes con una morbilidad neurológica alta en el postoperatorio inmediato que se reduce de forma significativa a las cuatro semanas. Los estudios en curso deben definir los márgenes de seguridad para la resección funcional que tengan en consideración el 'shift' cerebral operatorio. Finalmente, queda por demostrar el beneficio de estos protocolos en intervalo libre de enfermedad, de recidiva o en la supervivencia final de los pacientes.

PALABRAS CLAVE. Corteza cerebral motora. Neuronavegación. Tumor cerebral intrínseco. Monitorización neurofisiológica intraoperatoria. Craneotomía.

Treatment of intrinsic brain tumors located in motor eloquent areas. results of a protocol based in navegation, tractography and neurophysiological monitoring of cortical and subcortical structures 


\section{Summary}

Objectives. The role of the microsurgical management of intrinsic brain tumors is to maximize the volumetric resection of the tumoral tissue minimizing the postoperative morbidity. The purpose of our paper has been to study the benefits of an original protocol developed for the microsurgical treatment of tumors located in eloquent motor areas where the navigation and electrical stimulation of motor subcortical pathways have been implemented.

Materials and methods. A total of 17 patients operated on for resection of cortical or subcortical tumors in motor areas were included in the series. Preoperative planning for multimodal navigation was done integrating anatomic studies, motor functional MRI (f-MRI) and subcortical pathways volumes generated by diffusion tensor imaging (DTI). Intraoperative neuromonitorization included motor mapping by direct cortical and subcortical electrical stimulation (CS and $\mathrm{sCS}$ ) and localization of the central sulcus using cortical multipolar electrodes and the $\mathbf{N 2 0}$ wave inversion technique. The location of all cortical and subcortical stimulated points with positive motor response was stored in the navigator and correlated with the cortical or subcortical motor functional structures defined preoperatively.

Results. The mean tumoral volumetric resection was $89.1 \pm 14.2 \%$ of the preoperative volume, with a total resection $(\geq \mathbf{1 0 0 \%})$ in twelve patients. Preoperatively a total of $\mathbf{5 8 . 8 \%}$ of the patients had some motor deficit, increasing 24 hours after surgery to $76.5 \%$ and decreasing to $41.1 \%$ a month later. There was a great correlation between anatomic and functional data, both cortically and subcortically. However, in six cases it was not possible to identify the central sulcus and in many cases fMRI gave contradictory information. A total of 52 cortical points submitted to $\mathrm{CS}$ had positive motor response, with a positive correlation of $83.7 \%$. Also, a total of 55 subcortical points had positive motor response, being in these cases $7.3 \pm 3.1 \mathrm{~mm}$ the mean distance from the stimulated point to the subcortical tract.

Conclusions. The integration of preoperative and intraoperative anatomic and functional studies allows a safe functional resection of the brain tumors located in eloquent areas, compared to the tumoral resection based on anatomic imaging studies. Multimodal navigation allows the integration and correlation among preoperative and intraoperative anatomic and functional data. Cortical motor functional areas are anatomically and functionally located preoperatively thanks to MRI and fMRI and subcortical motor pathways with TDI and tractography. Intraoperative confirmation is done with CS and N20 inversion wave for cortical structures and with sCS for subcortical pathways. With this protocol we achieved a mean of $90 \%$ of volumetric resection in cortical and subcortical tumors located in eloquent motor areas with an increase of neurological deficits in the immediate postoperative period that significantly decreased one month later. Ongoing studies will define the safe limits for functional resection taking into account the intraoperative brain shift. Finally, it must be demonstrated if this protocol has any benefit for patients concerning disease free or everall survival.

KEY WORDS. Cerebral motor cortex. Neuronavigation. Intrinsic brain tumor. Intraoperative neurophysiological monitoring. Craniotomy.

\section{Introducción}

El papel de la cirugía en el tratamiento de los pacientes con tumores cerebrales intrínsecos continua siendo motivo de debate, aunque el mejor conocimiento de la biología de estos tumores y la incorporación de nuevos métodos de tratamiento oncológico están redefiniendo el papel de la cirugía ${ }^{3,4,14,20,21,26,27,33,38,41}$. El problema del tratamiento quirúrgico de los tumores cerebrales intrínsecos es el crecimiento infiltrante de todos ellos que imposibilita la delimitación preoperatoria de los límites tumorales para fijar los límites de la 'resección tumoral' oncológica. Un problema adicional es la expresividad clínica del tejido cerebral todavía viable funcionalmente pero infiltrado por el tumor, ya que su resección puede conducir a déficits neurológicos más o menos graves dependiendo de la elocuencia de la zona. Así se introduce el concepto de 'resección funcional', que implica que si bien es deseable que se maximice la 'resección tumoral', esta debe hacerse minimizando la probabilidad de secuelas neurológicas.

El planteamiento descrito nos obliga a desarrollar protocolos específicos de manejo de los tumores cerebrales intrínsecos ubicados en cada área elocuente relevante, especialmente las relacionadas con la actividad motora, verbal, visual y mnésica. En este trabajo vamos a describir el protocolo elaborado para el tratamiento de tumores intrínsecos en áreas elocuentes motoras y los resultados obtenidos en una serie de pacientes tratados siguiendo el mismo. El objetivo del protocolo es definir en la planificación preoperatoria el volumen tumoral y los volúmenes de las áreas corticales y de los tractos subcorticales funcionales motores. En la cirugía se localizan estas estructuras con navegación y se utilizan técnicas neurofisiológicas de ayuda para su validación anatómica y funcional, especialmente con monitorización neurofisiológica. Finalmente, se procede a la 'resección tumoral' con técnica microquirúrgica, que se extiende hasta los límites de 'resección funcional' 
que marca el estudio neurofisiológico.

\section{Material y métodos}

El estudio se ha realizado en una serie de pacientes con lesiones tumorales cerebrales intrínsecas superficiales o profundas localizadas en áreas elocuentes motoras y que fueron sometidos a tratamiento quirúrgico. En este sentido, se han considerado como áreas elocuentes motoras las siguientes zonas anatómicas: en el lóbulo frontal, el giro precentral (área motora primaria [AMP]; área 4 de Brodmann), la región posterior del giro frontal superior (área premotora [APM]; área 6 de Brodmann), la región anterior del giro frontal superior (área motora suplementaria [AMS]; área 8 de Brodmann) y la parte inferoposterior de la pars opercularis (AMP y área de Broca; áreas 44, 6 y 4 de Brodmann); en el lóbulo parietal, el giro postcentral (área somatosensorial [ASS]; áreas 3, 2 y 1 de Brodmann), que juega un papel importante en la preparación y control del movimiento; en la ínsula, el córtex insular (área 13 de Brodmann), que se considera una región importante en la planificación motora; $y$, por último, el ramillete de haces motores eferentes, somatosensitivos y de asociación que forman la cápsula interna en el espesor de la corona $\operatorname{radiata}^{4,7,10,36}$. Todos los pacientes han sido estudiados prospectivamente siguiendo el mismo protocolo básico de estudio clínico, de imagen, procedimiento quirúrgico y controles postoperatorios. En todos los casos se obtuvo el consentimiento informado de los pacientes para las diferentes técnicas de estudio, monitorización y tratamiento. El protocolo de estudio ha sido aprobado por el Comité de Ética del Hospital Clínico Universitario de Valencia, habiendo elaborado un Consentimiento Informado específico para la inclusión en el mismo.

Estudios y planificación preoperatoria. La parte inicial del protocolo consiste en confirmar por imagen la naturaleza tumoral de la lesión y sus características, así como obtener estudios anatómicos y funcionales que se fusionan en la estación de planificación del neuronavegador.

Los estudios de imagen para diagnóstico inicial incluían una TAC craneal en vacío seguido de un estudio de RNM con un protocolo de secuencias específico para 'caracterización tumoral, morfológica y volumétrica'. Este protocolo comprende las siguientes secuencias: 3D-T1 axial, 3D-MPRAGE sagital, T2 axial y coronal, FLAIR axial, perfusión, difusión, espectroscopía y T1 axial con gadolinio. Confirmada la sospecha de lesión tumoral por el estudio de imagen se realizaba una reconstrucción volumétrica tridimensional de la lesión en una estación de planificación Stealth ${ }^{\circledR}$ de Medtronic con la ayuda del paquete informático StealthViz ${ }^{\circledR}$. La reconstrucción tenía dos objetivos, el primero la determinación del volumen tumoral y el segundo la confirmación de la localización anatómica de la lesión en un área motora. Se definió como volumen tumoral en las lesiones con captación masiva, el volumen que ganaba contraste en los cortes T1 con gadolinio, mientras que en las lesiones sin captación masiva se consideró el volumen tumoral el área de hiperintensidad en la secuencia potenciada en FLAIR ${ }^{17,34,35,40,46}$. Para la localización anatómica se tomó como referencia el surco central en los estudios 3DT1 axial, identificado directamente o, en caso de distorsión por la patología subyacente, localizado con la ayuda del ramo ascendente del surco cingulado que marca el borde anterior del lóbulo precentral y que es bien visible en el corte sagital de línea media ${ }^{4,7,10,36}$. Las lesiones se agruparon según la clasificación propuesta por Sawaya et al. ${ }^{39}$ en base a su localización relativa a la función cerebral: grado I, lesión en área no elocuente; grado II, lesión cercana a área elocuente; y grado III, lesión en área elocuente.

El estudio anatómico preoperatorio se completaba con un estudio de RNM de tensor de difusión (TDI), elaborándose una tractografía de los haces implicados en cada caso, en especial, la vía corticoespinal, fascículo longitudinal superior y fascículo uncinado ${ }^{10}$. Gracias a software de distribución libre (MRIcro, DTV.II SR VolumeOne, MedINRIA, Image J) los tractos seleccionados se convertían en volúmenes que se montaban sobre el estudio anatómico. Como estudio funcional preoperatorio y con la finalidad de correlacionar las áreas anatómicas corticales teóricamente elocuentes con su función motora específica, se realizó un estudio de RNM-f con paradigmas de estudio de función motora (pie, mano y lengua) ${ }^{2}$, que fue montado sobre un estudio de RNM anatómico.

Todos los estudios de resonancia magnética se realizaron en las instalaciones de ERESA en el Hospital Clínico Universitario de Valencia y con las máquinas MAGNETOM Trio de 3 Teslas de Siemens (Erlangen, Germany) y Signa Excite de 3 Teslas (General Electrics Health) y los de RNM funcional con la colaboración del Departamento de Psicología de la UJI de Castellón. Gracias a una conexión en red entre las máquinas de resonancia, la estación de planificación y el neuronavegador intraoperatorio todos los estudios de imagen referidos se enviaban directamente desde los equipos de estudio hasta la estación de planificación StealthStation de Medtronic ubicada en el Servicio de Neurocirugía, donde se realizó la fusión y posterior planificación. Como base para la fusión se eligió, en función del patrón de captación, el estudio 3D T1 con gadolinio o el FLAIR sobre el que se fusionaba el estudio de RNM-f y los volúmenes de los tractos generados a partir de la tractografía (fig. 1 y 2). Tras la fusión se realizó la planificación preoperatoria, que era enviada directamente de nuevo vía intranet al neuronavegador situado en el quirófano de Neurocirugía.

Ayudas intraoperatorias. Durante la cirugía se dispuso de una serie de técnicas de confirmación anatómica 


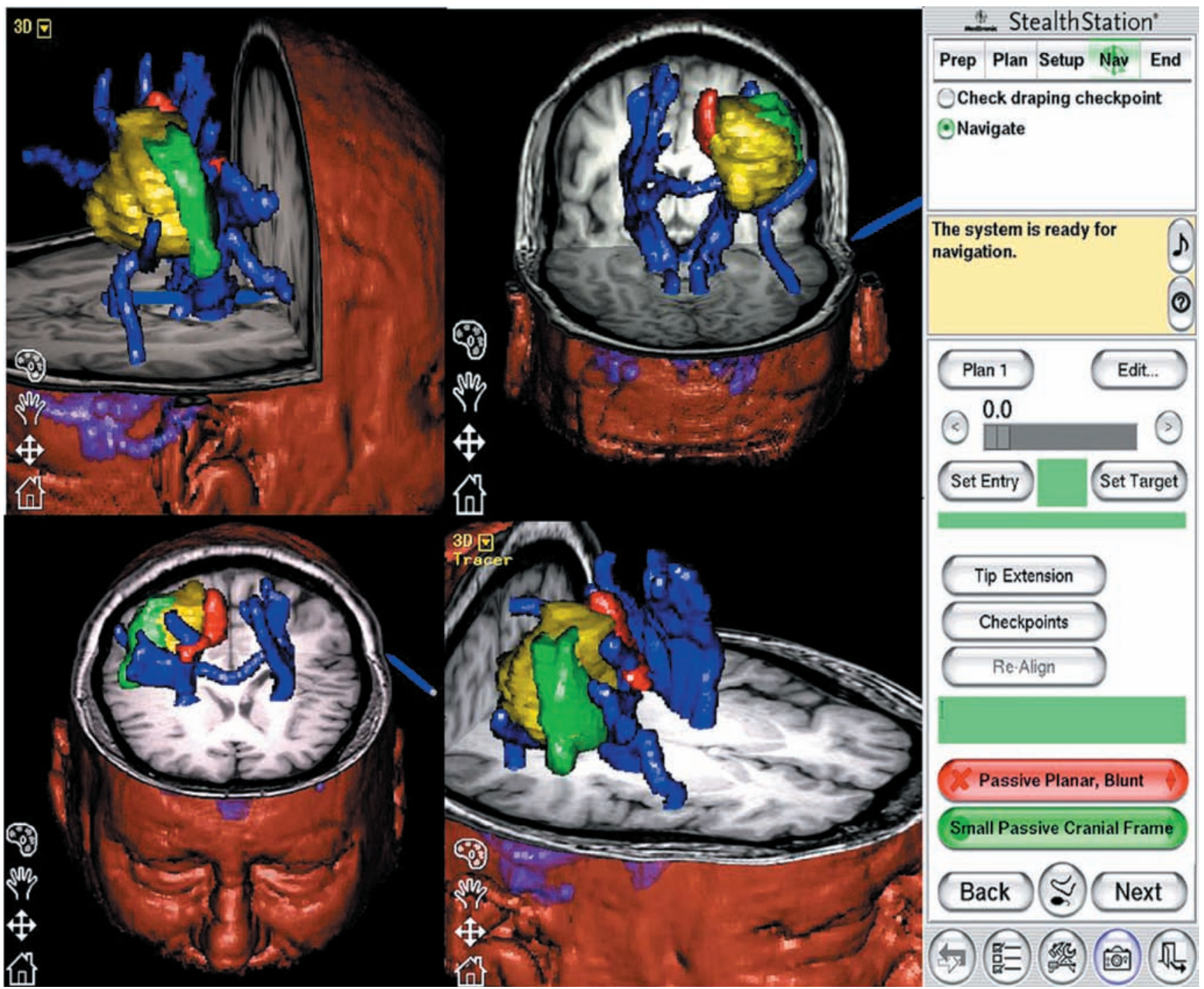

Figura 1. Resultado de la fusión de los estudios preoperatorios para la planificación preoperatoria. Se visualizan los volúmenes del tumor (amarillo), áreas corticales funcionales correspondientes a la mano (verde) y pie (rojo) contralaterales en el estudio de RNM-f y haz corticoespinal piramidal (azul), en un caso de tumor frontal derecho.

y funcional. La confirmación anatómica se basaba en los datos proporcionados por el reconocimiento por parte del cirujano de la neuroanatomía de los giros y surcos de la corteza cerebral y, especialmente, por la identificación gracias al neuronavegador de esas mismas estructuras u otras de referencia como vasos o, eventualmente, del tumor en casos en los que afloraba a la superficie cerebral. Generalmente se utilizó el módulo de navegación electromagnético por su mayor versatilidad y comodidad para su uso con el microscopio quirúrgico (Pentero ${ }^{\circledR}$ Zeiss) y en ausencia del interface de navegación en el mismo. De esta forma, la navegación permitía tanto la localización de estructuras anatómicas (surcos y giros corticales, vasos visibles, volumen tumoral, volumen de los tractos subcorticales) como funcionales (volumen de activación funcional en la RNM-f de las diferentes tareas motoras).

La ayuda funcional intraoperatoria se basa en la monitorización neurofisiológica, que comprendía tres técnicas ${ }^{6,9,28,29}$. Inmediatamente después de la craneotomía y la localización anatómica de las estructuras con el navegador se procedía al mapeo cortical con estimulación cortical directa (Equipo Neuropack ${ }^{\circledR}$ de Nihon Koden y Estimulador Externo Ojemann ${ }^{\circledR}$ OCS2 de Integra NeuroSciences) identificando de esta forma las áreas corticales con respuesta motora, que eran señaladas en el campo quirúrgico con marcas sobre el córtex (ECD: mapeo del área motora cortical por estimulación bipolar directa y registro electromiográfico). En caso de lesiones alrededor 


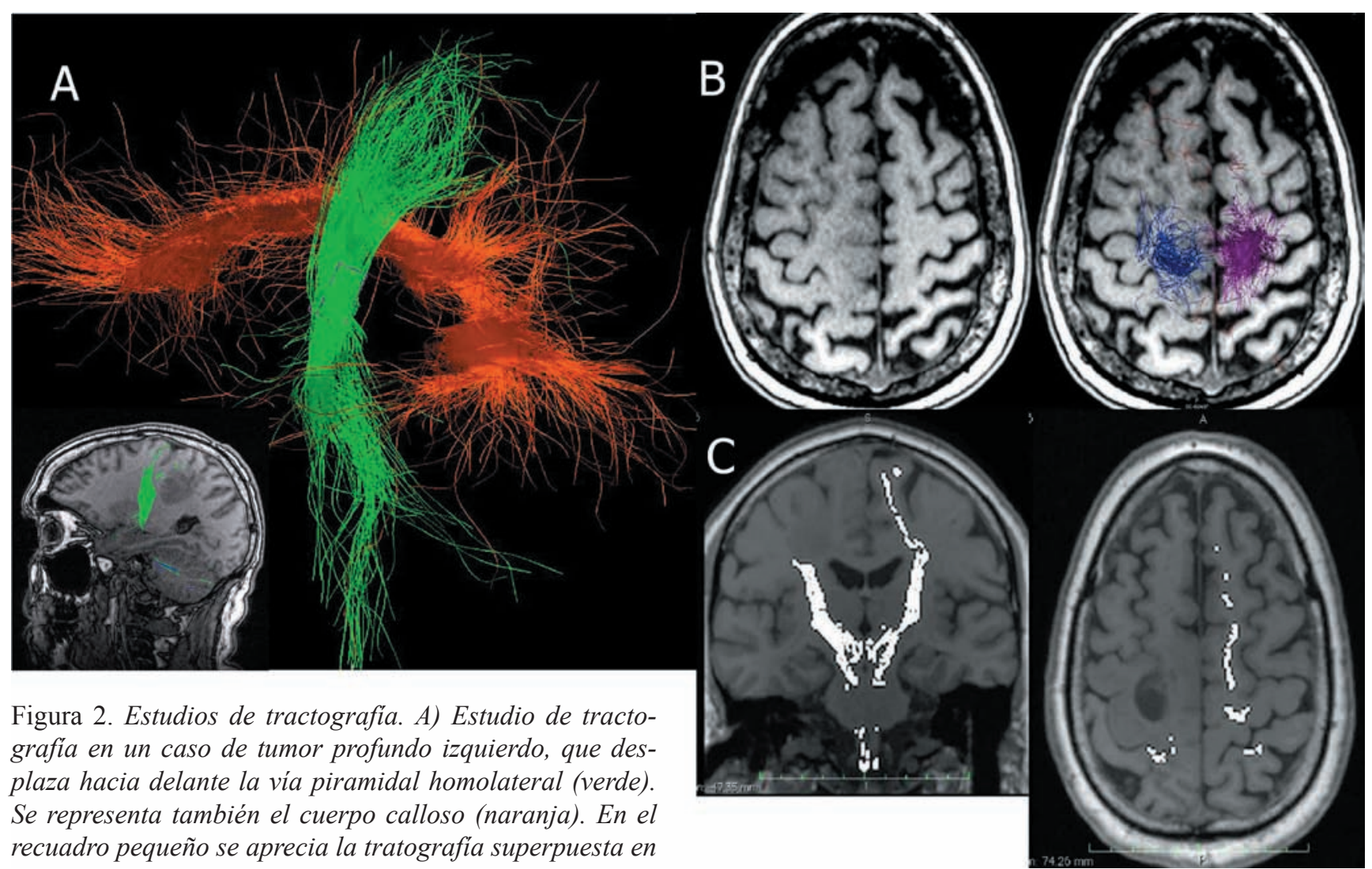

un estudio morfológico en el que se aprecia la lesión. B) Estudio semejante al anterior, donde se aprecia el origen de la vía piramidal en el giro precentral en un caso de tumor situado en el lado izquierdo. En el corte axial de la izquierda se aprecia la asimetría de ambos giros con el engrosamiento y rarefacción del giro prefrontal izquierdo. En el corte axial de la derecha se han superpuesto las fibras emergentes de ambos lados, apreciándose un menor número y mayor dispersión de las fibras del lado del tumor. C) Volúmenes de la vía corticoespinal después de ser fusionados con un estudio anatómico y listo para la navegación, en un caso de tumor situado en el giro prefrontal izquierdo. Se aprecia la asimetría entre el lado íntegro y el que aloja el tumor en un corte coronal y otroaxial.

del surco central, éste era identificado con un electrodo plano de seis contactos y la técnica de inversión de onda N20 (N20: mapeo del surco central con potenciales evocados somatosensoriales del nervio mediano contralateral). Finalmente, tras la exéresis volumétrica lesional se procedía a la estimulación directa de los tractos subcorticales (EsCD: mapeo motor subcortical por estimulación bipolar directa y registro electromiográfico). Como control se realizaba estimulación transcraneal del córtex motor homo y contralateral con registro electromiográfico y el registro de potenciales corticales evocados somatosensoriales (PESS) homo y contralaterales por estimulación del nervio mediano y tibial posterior (Estimulador Digitimer D185® y equipo Neuropack $\left.{ }^{\circledR}\right)$. También se realizaba un registro electrocorticográfico continuo con el mismo electrodo plano usado para identificar el surco central, que se dejaba alojado subduralmente mas allá de la craneotomía, y dirigido siempre frontalmente.

Resección tumoral. Todos los pacientes fueron inter- venidos bajo anestesia general y en ausencia de relajación neuromuscular para permitir la monitorización neurofisiológica intraoperatoria. Se diseñaron craneotomías amplias centradas sobre la lesión localizada con el navegador, incluyendo además el área cortical elocuente motivo de estudio intraoperatorio. La lesión era extirpada con ayuda del microscopio quirúrgico, abordaje transulcal y técnicas microquirúrgicas estándar, incluyendo uso libre de aspirador ultrasónico, técnicas de fluorescencia tras administración de 5-ALA ${ }^{42}$ y aplicación de obleas de gliadel en las lesiones de alto grado en los márgenes de la cavidad $^{44}$. El objetivo de la cirugía era la exéresis completa de la lesión definida en la planificación preoperatoria como tumor ('resección tumoral'), con el margen de tejido más amplio posible sin provocar nuevos déficits neurológicos ('resección funcional'). La combinación de la información anatómica proporcionada por la neuroanatomía y la navegación y la funcional proporcionada por la RNM-f y el mapeo cortical con ECD permitía el diseño del abordaje 


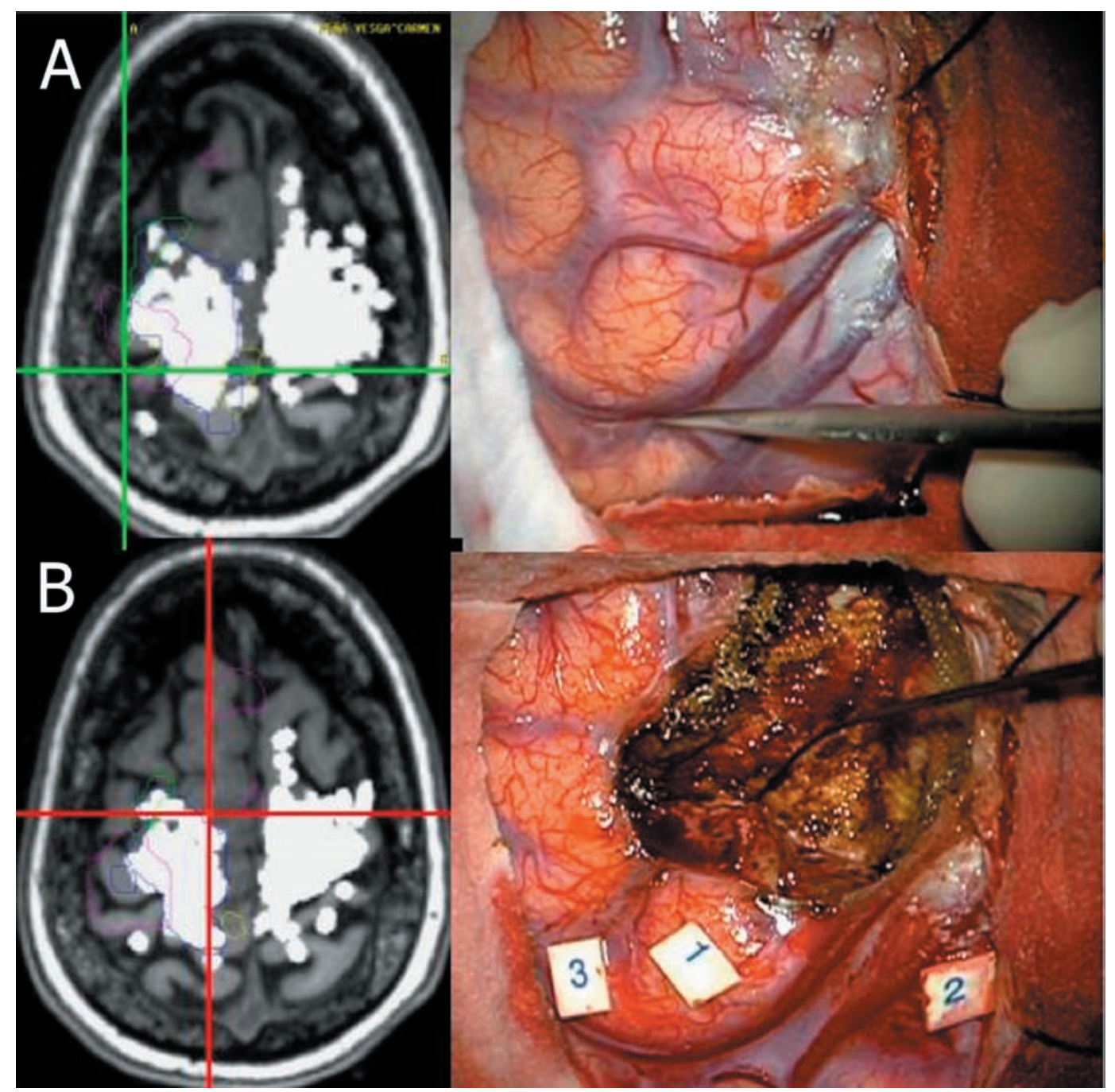

Figura 3. Imágenes intraoperatorias de la navegación y del campo quirúrgico en un caso de tumor localizado en la parte posterior del giro frontal superior izquierdo. A) Momento de la identificación del surco central con el puntero del navegador e imagen de la navegación de dicho punto sobre un corte anatómico axial donde se ha resaltado el volumen de la vía piramidal. B) Imagen quirúrgica tras la resección volumétrica tumoral en el momento de la estimulación eléctrica subcortical (EsCD), donde se aprecian sobre el giro central las marcas de los puntos con respuesta motora en la estimulación eléctrica cortical (ECD) sobre un corte anatómico axial con el volumen de la vía piramidal, indicando la navegación que se está estimulando el límite anterior de dicha estructura.

transulcal o, en su caso de la cortectomía, para el abordaje y exéresis volumétrica de la lesión (fig. 3). La información anatómica de la resección volumétrica calculada proporcionada por el navegador, tomando en consideración la posición de los haces subcorticales (volúmenes generados por la tractografía), junto al resultado en su caso del mapeo de la sustancia blanca con EsCD, marcaban el límite de resección subcortical. En cualquier momento del procedimiento, sin embargo, la decisión operativa aceptando el resultado de la planificación o, en caso de conflicto, de las modificaciones pertinentes, era responsabilidad final del cirujano responsable.
Valoración postoperatoria. Para la valoración de los resultados se obtuvieron datos clínicos, de imagen y de correlación anatomo-funcional. La anestesia era revertida en el propio quirófano o en la Unidad de Reanimación unas seis horas más tarde. Aparte de los controles neurológicos rutinarios evolutivos, se efectuó una valoración neurológica por protocolo a las 24 horas y a los 30 días de la cirugía, en la que se recogían los nuevos déficits neurológicos y la evolución de los presentes en la exploración previa. Aparte de los estudios rutinarios de TAC postoperatorio para control de la cirugía, se realizaba antes de las 72 horas una RNM para 'control de resección', con secuencias axial 
T1 con gadolinio, axial FLAIR y difusión. Sobre estos estudios, tras un nuevo análisis volumétrico, se realizó el cálculo del grado de exéresis tumoral, definiendo cuatro grupos: exéresis total ( $\geq 100 \%)$; subtotal $(90-99 \%)$; parcial (50-89\%) y sub-óptima $(<50 \%)$, según la clasificación propuesta por Berger et $\mathrm{al}^{3}$. Finalmente, se recogieron los datos proporcionados por el estudio anatomopatológico.

Además, durante la cirugía la posición de todos los puntos donde se obtenía una respuesta motora positiva a la ECD o EsCD eran marcados con el puntero y sus coordenadas registradas mediante el navegador, con el objeto de analizar el grado de correlación entre la estimulación, referencias anatómicas corticales y sulcales, tracto anatómico y área cortical funcional identificada en la RNM-f. En la EsCD se calculaba en el navegador la distancia entre el punto de respuesta a la estimulación y el tracto anatómico estimulado (fig. 3B). Se consideraba que existía 'buena correlación' entre los estudios en caso de que la respuesta motora con la ECD o EsCD se obtuviera en la zona anatómica cortical o subcortical que se había considerado como funcional.

\section{Resultados}

Se han incluido en el estudio un total de 17 pacientes intervenidos quirúrgicamente entre junio de 2008 y abril de 2009 en el Servicio de Neurocirugía del Hospital Clínico Universitario de Valencia, con tumores en áreas motoras elocuentes. La serie estaba compuesta por 7 mujeres y 10 hombres (1:1.4) con una edad media ( \pm desviación standard) de $54 \pm 9.5$ años. La clínica principal de presentación fue irritativa en once pacientes $(64.7 \%)$, deficitaria en cinco pacientes $(29.4 \%)$ y en el paciente restante (5,9\%) el síntoma de alarma fue cefalea refractaria a tratamiento analgésico. Todos los pacientes recibieron tratamiento sintomático, anticomicial o con corticoides antes de la intervención, con control o mejoría de los síntomas. En el momento de la cirugía 10 pacientes $(58.8 \%)$ presentaban algún tipo de déficit motor en la exploración neurológica. Como antecedente de interés destaca que dos pacientes habían sido intervenidos previamente de un oligodendroglioma GII, presentando ahora una recidiva local.

El volumen tumoral medio era de $27.2 \pm 21 \mathrm{cc}$ (rango 3.4-72.7cc). Respecto a la localización anatómica de las lesiones, en 12 pacientes $(70.6 \%)$ era cortical o subcortical (3 giro postcentral; 4 giro precentral; 2 parte posterior giro frontal superior; 1 parte anterior giro frontal superior; 2 pars opercularis) y en los cinco restantes (29.4\%) era profunda ( 3 en corona radiata desplazando la cápsula interna; 2 en sustancia blanca insular). La distribución de las lesiones según la clasificación de Sawaya et al. ${ }^{39}$ era la siguiente: siete lesiones grado II (41.2\%; cercana a área elocuente) y diez grado III (58.8\%; en área elocuente). La RNM-f se realizó en 11 pacientes y mostraba en todos los casos áreas de activación sobre el área precentral contralateral al hemicuerpo examinado. En tres pacientes se obtuvieron áreas de activación positivas superpuestas en mayor o menor grado a la lesión, siempre gliomas de bajo grado en área precentral. El estudio de RNM con tensor de difusión fue completado en todos los casos. La tractografía se realizó sin inconvenientes, y se segmentaron e individualizaron la vía corticoespinal en todos los estudios, fascículo longitudinal superior en ocho y el fascículo uncinado en dos (fig. 2A).

La fusión de los estudios anatómicos y de diagnóstico, de RNM-f y tractografía para la planificación preoperatoria y la navegación se consiguió en todos los casos con precisión elevada (error estimado inferior a $1.5 \mathrm{~mm}$ ), la mayor parte de las veces de forma automática y cuando esto no fue posible por puntos, para alcanzar el mismo nivel de precisión (fig. 1).

Resultados clínicos. Todas las lesiones fueron localizadas en la cirugía y confirmadas anatomopatológicamente. No hubo mortalidad intra ni postoperatoria, ni tampoco morbilidad relacionada con los métodos de estudio o monitorización, salvo que en cuatro pacientes se produjeron una o más crisis convulsivas clínicas o electroencefalográficas intraoperatorias relacionadas con la ECD. El estudio anatomopatológico demostró las siguientes lesiones: astrocitoma de bajo grado ( 3 casos), astrocitoma anaplásico ( 3 casos), oligoastrocitoma ( 2 casos), oligodendroglioma de bajo grado ( 2 casos), glioblastoma multiforme (4 casos) y metástasis ( 3 casos). A las 24 horas de la cirugía había focalidad neurológica motora deficitaria en $13(76.5 \%)$ de los pacientes intervenidos, que se redujo sustancialmente a los 30 días tanto cuantitativamente (7 pacientes, $41.1 \%$ ) como cualitativamente, toda vez que en este momento sólo afectaba a las actividades de vida diaria de un paciente con monoparesia del miembro superior derecho, ya que en el resto eran de grado mínimo o bien toleradas. El análisis volumétrico postoperatorio mostró un grado de exéresis tumoral del $89.1 \pm 14.2 \%$ del volumen tumoral calculado en los estudios preoperatorios, con resección total en ocho pacientes (47.1\%), subtotal en tres $(17.6 \%)$ y parcial en los seis restantes $(35.3 \%)$. La resección media era mayor en los tumores de localización en los opérculos frontales del giro frontal inferior, giro frontal superior y corona radiata, mientras que fue menor en los insulares y de giros pre y postcentral. En cuanto a la correlación del porcentaje de resección con los grados Sawaya $^{39}$, nos encontramos con que en los tumores de Grado II el grado de resección fue del 91.9\%, mientras que en los Grado III el grado de resección medio fue de $81.1 \%$. Respecto a la naturaleza de la lesión, el grado de resección fue máximo en metástasis y oligodendrogliomas, menor en astrocitomas anaplásicos y glioblastomas y menor todavía 
en los astrocitomas de bajo grado.

Correlación anatomo-funcional. Mediante referencias anatómicas y con neuronavegación se localizó de forma segura el surco central y consecuentemente el giro precentral en 11 estudios (64.7\%), mientras que en los seis casos restantes la identificación directa del surco central fue imposible por la distorsión debida al tumor (fig. 3A). La RNM-f mostraba siempre activación sobre el giro precentral localizado anatómicamente, aunque en muchos casos el grado de seguridad no era alto dado que había actividad sobre el tumor y en otros muchos había zonas de activación dispersas por delante o detrás del giro precentral. En todos los casos donde se definió la vía corticoespinal mediante la tractografía hubo una total correlación con el origen de la misma en el giro precentral definido en los estudios anatómicos de imagen, incluyendo los casos donde las lesiones habían desplazado más o menos groseramente el giro precentral donde se origina dicho tracto o la propia vía corticoespinal, lo que fue de gran ayuda para confirmar la localización anatómica del giro precentral (fig. 2). La distancia media entre la lesión y el tracto corticoespinal en los tres casos de tumores subcorticales en la corona radiata fue de $9.3 \mathrm{~mm}(2.2 ; 12.1 ; 13.5 \mathrm{~mm})$.

En los tumores localizados en giro postcentral, giro precentral y en el giro frontal superior se realizaron un total de 52 ECD con respuesta motora positiva que identificaba el área motora primaria, alcanzándose una correlación positiva del $83.7 \%$ entre dichos puntos registrados en el neuronavegador y las áreas funcionales correspondientes identificadas en la RNM-f. En seis casos de tumores vecinos al surco central se tuvo que utilizar la técnica de inversión de onda N20 para localizar el surco central debido a la distorsión anatómica producida por el tumor que impedía reconocerlo con seguridad con referencias neuroanatómicas ni con navegación. En cinco de estos casos la localización del surco central resultó congruente con los volúmenes del tracto corticoespinal identificados con TDI (fig. 2C). En el caso restante el surco central localizado con la inversión de onda N20 estaba $4 \mathrm{~mm}$ por detrás del surco anatómico identificado por neuronavegación y visible en la corteza, siendo tomado en este caso el surco central anatómico como referencia. La navegación con las imágenes obtenidas con la tractografía resultó de especial ayuda en la resección de lesiones cercanas a la vía corticoespinal, siendo en estos casos el elemento de referencia que determinó la extensión de la resección en dirección a dicho tracto. Como se ha indicado más arriba, tras la resección volumétrica del tumor guiada por navegación y estimulación cortical, se realizaba la estimulación del lecho tumoral para identificar así los tractos motores subcorticales y, en 15 pacientes, se realizaron un total de $55 \mathrm{EsCD}$ con respuesta motora positiva que identificaban tractos corticoespinales procedentes del área motora primaria (fig. 3B). En estos casos, la dis- tancia media entre los puntos de respuesta y la ubicación de los haces en el navegador era de $7.3 \pm 3.1 \mathrm{~mm}$ (rango 1.8-13.4mm). Dado que los parámetros de la ECD y EsCD influyen en la obtención de una respuesta, se empleaban estímulos de $0.5-1 \mathrm{~ms}$ de duración y con intensidades entre 2-10mA. Los PESS sólo se alteraron en un caso de lesión profunda adyacente a la vía piramidal en el espesor de la corona radiata. Cuando se analiza la relación existente entre la focalidad neurológica a los 30 días y la distancia entre el último punto con respuesta positiva a la estimulación subcortical y el tracto corticoespinal, se aprecia que hay una relación inversa entre ambas, con un margen empírico de seguridad a partir de $8-10 \mathrm{~mm}$.

\section{Discusión}

La recomendación actual en el tratamiento de los tumores cerebrales intrínsecos es la resección microquirúrgica radical de los mismos manteniendo la integridad de la función neurológica del paciente, seguido de la observación por neuroimagen o del tratamiento oncológico según el tipo y grado tumoral. Sin embargo, este límite de 'resección tumoral' debe extenderse sólo hasta el punto en que como consecuencia de la resección tisular pueda aparecer un deterioro de la función neurológica, lo que constituye el límite de 'resección funcional'. Este planteamiento es útil tanto en caso de tumores de alto como de bajo grado. En los primeros es recomendable mantener la función neurológica dado que el pronóstico vital de los pacientes continua siendo muy pobre a pesar del tratamiento oncológico agresivo multimodal, por lo que debe cuidarse la calidad de vida en el periodo de supervivencia. En los gliomas de bajo grado también es recomendable mantener íntegra la función neurológica, ya que la clínica inicial suele ser paucisintomática, bien controlada con medicación y la supervivencia es más larga. Además, recientemente, se ha demostrado que en estos pacientes es posible incluso plantearse resecciones subtotales para conservar funciones alojadas en áreas infiltradas por el tumor, ya que con el tiempo pueden reubicarse alejadas ante el crecimiento tumoral ${ }^{13}$.

En la práctica clínica hay una gran cantidad de técnicas que sirven para delimitar anatómica y funcionalmente las áreas funcionales corticales y subcorticales y hacer más segura la cirugía de los tumores ubicados en las mismas o en su proximidad. Una de las actividades funcionales más crítica y elocuente es la motora, por lo que el tratamiento microquirúrgico de los tumores de dicha zona presenta particular interés. Las áreas motoras y asociativas motoras comprenden el giro postcentral, surco central, giro precentral, giro frontal superior y opérculos frontales, a las que hay que añadir las conexiones subcorticales cortas entre los giros vecinos (fascículos en 'u') y las largas, como el fascículo longitudinal superior, el uncinado, y fundamen- 
talmente la vía corticoespinal efectora ${ }^{4,7,10,36}$.

Protocolo clínico. En nuestro estudio, las áreas corticales se han determinado anatómicamente con técnicas de imagen, concretamente con reconstrucciones tridimensionales de imágenes de RNM potenciadas en T1. Esta identificación anatómica es habitualmente segura, y aunque puede estar distorsionada por la propia patología subyacente, se determina preoperatoriamente y se comprueba intraoperatoriamente con navegación, correlacionándola con la neuroanatomía del campo microquirúrgico. Técnicas alternativas de localización anatómica sin ayuda de navegador, como son las técnicas craniométricas ${ }^{37}$, ofrecen una fiabilidad sustancialmente inferior y son inadecuadas para una cirugía segura en lesiones pequeñas, subcorticales o profundas. La posibilidad de localizar los haces subcorticales en los estudios preoperatorios mediante TDI y tractografía ha supuesto un nuevo avance en el conocimiento de las relaciones anatómicas de las lesiones con dichas vías, ya que permiten estudiar la disposición de los haces de la sustancia blanca de forma tridimensional voxel a voxel a lo largo de todo el cerebro humano ${ }^{4,18,30,31,32,45}$. Esta ha sido la razón de nuestro interés en incorporar la TDI al sistema de navegación y así reconocer la situación anatómica de los puntos sometidos a EsCD para tomar decisiones en cuanto a continuar con la resección tumoral. Hasta ahora, la EsCD de los márgenes de resección permitía al cirujano reconocer la integridad funcional de las fibras estimuladas ${ }^{6,9,28,29}$, pero sin tener una idea clara del tracto estimulado ni de la situación espacial del mismo en relación al punto estimulado. En nuestra experiencia, la incorporación de las TDI al sistema de navegación StealthStation de Medtronic ha sido posible de forma sencilla aunque muy laboriosa, con lo que ha aumentado de forma significativa el trabajo preoperatorio de planificación de la cirugía a cambio de un mejor conocimiento anatómico de las vías anatómicas subcorticales y de la propia lesión. Hasta este momento, nuestro protocolo de planificación preoperatoria y monitorización intraoperatoria se centraba en la identificación de la lesión, de los surcos, giros y determinadas estructuras vasculares corticales de referencia anatómica y al conocimiento de la funcionalidad de porciones corticales elocuentes. La incorporación de la tractografía y de la EsCD nos ha permitido conocer la situación anatómica de los tractos de la sustancia blanca y estudiarlos funcionalmente, más allá de la intuición e imaginación del cirujano.

La RNM-f permite asignar preoperatoriamente una determinada función a una zona cortical que se activa como consecuencia del desarrollo de una actividad elocuente excitada a través de un paradigma de estudio específico y reproducible ${ }^{2}$. La fusión de la RNM-f con los estudios anatómicos realizada en nuestro protocolo permite incorporar esta información a la neuronavegación intraoperatoria ${ }^{1,16,22,28}$. Sin embargo, en nuestra experiencia las respuestas motoras a la ECD no son superponibles funcionalmente a las áreas activadas en la RNM-f, ya que este último estudio se hace con actividades que, aunque sencillas, involucran varios grupos musculares con representación cortical mucho más amplia que la que corresponde a la de un sólo músculo registrado con la ECD. Esto ha hecho que nuestra confianza en la RNM-f haya decaído con la mayor experiencia clínica y actualmente pensamos que no aporta beneficios frente a la ECD. Aunque no tenemos experiencia clínica con la estimulación magnética transcraneal u otros sistemas de localización funcional preoperatoria ${ }^{24,25,43}$, la ECD ha confirmado en nuestra experiencia una gran fiabilidad, versatilidad y reproducibilidad frente a los estudios preoperatorios.

Así, globalmente, la monitorización neurofisiológica ha sido una gran ayuda intraoperatoria de localización anatómica y funcional. La técnica de inversión de la onda N20 se basa en el registro con un electrodo multicontacto de los PESS tras estimulación de un nervio periférico de forma que el surco central se identifica entre el par de electrodos en los que se produce el cambio de polaridad de la onda N20. Esta técnica permite identificar el surco central en casos de distorsión anatómica producida por la lesión cuando resulta imposible o insegura de reconocer dicha estructura con referencias neuroanatómicas o con navegación. También la EsCD nos permite confirmar anatómicamente la topografía de la vía piramidal y confrontarla a los datos proporcionados por la navegación directa de la misma. En este sentido, nuestra experiencia demuestra que existe una distancia de 8-10mm entre el punto de estimulación de la vía piramidal con respuesta motora positiva y dicha estructura identificada por tractografía e identificada por navegación que marca el límite de seguridad de la resección funcional vecina al haz corticoespinal, que viene a ser semejante a la propuesta por otros autores ${ }^{5,30,31}$. Esta distancia está influida por los parámetros de estimulación, pero por debajo de la misma se producen déficits neurológicos. Finalmente, la EDC permite un buen correlato anatomo-funcional de la corteza motora del giro precentral. Sin embargo, en pacientes anestesiados como sucede en nuestro protocolo, la ECD carece de utilidad en otras zonas elocuentes, ya que lamentablemente no se pueden obtener datos relacionados con la función verbal o con funciones motoras muy típicas de áreas funcionales en opérculos, ínsula o en área precentral o suplementaria motora frontal ${ }^{6,9,11}$.

El aspecto más novedoso de nuestro protocolo ha sido la incorporación de la navegación de los tracto subcorticales, concretamente la vía corticoespinal, gracias a la tractografía. Hay que resaltar que el procesado de las TDI y su conversión en formato DICOM y/o Analyze que permite la fusión en el navegador con las imágenes anatómicas y funcionales de RNM consume una importante 
cantidad de tiempo en la planificación preoperatoria. Sin embargo, la información proporcionada por la TDI permite acortar el tiempo en el proceso intraoperatorio de localización funcional cortical con ECD e inversión de onda N20 para definir abordaje transulcal al tumor. Además, una vez realizada la resección volumétrica tumoral, el conocimiento anatómico y la capacidad de visualizar mediante navegación las vías subcorticales y, en especial la corticoespinal, reduce el número de estimulaciones subcorticales para la localización de las mismas y mejora la interpretación de la información obtenida a la hora de maximizar la resección.

Resultados clínicos. El objetivo inmediato del protocolo es maximizar la resección de las lesiones de la zona y minimizar la morbilidad. El análisis de los resultados de nuestra experiencia clínica muestra que la integración de la tractografía a la navegación ha aumentado sustancialmente el grado de resección de los tumores de áreas motoras y que, al mismo tiempo, se producen más déficits neurológicos. Efectivamente, el grado de 'resección tumoral' se encuentra en el $90 \%$ del volumen total de la lesión, con una exéresis total en más de la mitad de la serie y, al mismo tiempo, casi todos los pacientes tienen en el postoperatorio inmediato nuevos déficits neurológicos o un empeoramiento de los ya existentes. Afortunadamente, los déficits neurológicos son reversibles total o parcialmente en la mayor parte de los pacientes y al cabo de 30 días sólo un paciente presentaba problemas que le dificultaban llevar a cabo sus actividades diarias. La literatura permite reconocer la presencia de elevadas tasas de focalidad neurológica en el postoperatorio precoz de lesiones en áreas elocuentes y su resolución posterior $^{9,11,39}$. Esto se ha relacionado con el hecho de que la seguridad que proporciona al cirujano la monitorización anatómica y funcional le invitaría a trabajar muy cerca de las áreas elocuentes, con lo que los inevitables fenómenos de tracción, calor por termocoagulación bipolar, edema citotóxico o fenómenos de reorganización microvascular tendrían una mayor repercusión funcional durante el postoperatorio inmediato. Ha quedado fuera de nuestro estudio, dado el diseño del mismo y el tamaño muestral, identificar si el protocolo aumenta el periodo libre de enfermedad o la supervivencia.

Limitaciones de las técnicas y del protocolo. El protocolo propuesto se basa en disponer de las técnicas preoperatorias e intraoperatorias de localización anatómica y funcional para seleccionar de entre ellas las más eficientes para cumplir sus objetivos específicos según la función involucrada en el procedimiento. Los estudios preoperatorios actualmente disponibles comprenden estudios de imagen como la tomografía axial computada (TAC), imagen por resonancia nuclear magnética (RNM), tomografía de emisión de positrones (PET) y tomografía computada de emisión de protones (SPECT) y todos ellos presentan como principal limitación la dificultad de definir
2011; 22: 23-35

los límites lesionales debido al carácter infiltrativo del crecimiento de este tipo de tumores ${ }^{8,12}$. La capacidad de detección de los tumores con técnicas de imagen se relaciona con el llamado 'índice de invisibilidad' definido por el cociente $\mathrm{D} / \mathrm{p}$, donde ' $\mathrm{D}$ ' representa la capacidad de difusión o invasión del tumor medida en $\mathrm{mm} /$ día y que depende del tumor y de las características del sustrato anatómico, mientras que 'p' representa la capacidad de proliferación del tumor medido en tiempo de duplicación y que depende del tumor. Cuando el índice D/p es alto nos encontramos ante un tumor muy infiltrante y difuso, difícilmente detectable con técnicas de imagen; por el contrario, sin el índice D/p es bajo se trata de un tumor con alto patrón proliferativo y por lo tanto muy compacto y fácilmente detectable con las técnicas de imagen ${ }^{15}$. Por ello, no hay más remedio que convenir que para definir el volumen tumoral se considera la zona de cambio de señal en FLAIR para los tumores altamente infiltrantes ('invisibles') y la zona de captación de gadolinio en T1 para los tumores altamente proliferativos ('visibles') y esperar que nuevos medios diagnósticos permitan mejorar este aspecto ${ }^{46}$. En lo que se refiere al reconocimiento estricto de estructuras anatómicas de referencia, la RNM en secuencia multicorte T1 para reconstrucción 3D es adecuada para identificar surcos, giros y algunas estructuras anatómicas vasculares, ya que la distorsión de imagen intrínseca al estudio de imagen por resonancia no introduce errores apreciables. Algunos detalles anatómicos pueden ser más fácilmente reconocibles en las secuencias T2. Sin embargo, las estructuras subcorticales sólo se pueden identificar mediante estudio de DTI y tractografía.

Los estudios funcionales preoperatorios vinculan acti vidades a determinadas áreas corticales y se fusionan en estudios de imagen de RNM anatómicos. Hay disponibles diversos medios de estudio, que ofrecen diferentes visiones del problema y con sus ventajas e inconvenientes: RNM funcional (RNM-f), imágenes de origen electromagnético (Electromagnetic Source Imaging (ESI): magnetoencefalografía y electroencefalografía), tomografía de emisión de positrones (PET) y estimulación eléctrica transcraneal (TMS) 4,19,24,25,43. La selección de este tipo de técnicas depende fundamentalmente de la disponibilidad, ya que se trata de equipos costosos y escasos. Por esta causa, hemos desarrollado en nuestro medio la RNM-f que, aún a costa de ser menos exacta, es muy versátil a la hora de explorar diferentes funciones con paradigmas muy diversos ${ }^{2}$.

Las ayudas intraoperatorias de localización anatómica se basan en el uso de referencias craniométricas, neuroanatomía de giros y surcos y localización estereotáctica con marco o sin marco (neuronavegación) y las técnicas de imagen intraoperatoria con ultrasonidos, rayos $\mathrm{X}$ o resonancia magnética ${ }^{4}$. La localización estereotáctica sin marco mediante sistemas de neuronavegación óptica o electromag- 
nética ha sido el método utilizado en nuestro protocolo, ya que permite la planificación preoperatoria más cuidadosa y la fusión de todos los estudios preoperatorios anatómicos y funcionales. Sin embargo, la navegación se basa en el uso durante el acto quirúrgico de estudios realizados al paciente en el periodo preoperatorio, y está aceptado que durante la cirugía se producen indefectiblemente distorsiones volumétricas que pueden ser trascendentes afectando a la fiabilidad de la localización de estructuras. Este fenómeno se conoce como 'brain shift', está producido por factores como la entrada de aire o salida de LCR del espacio subaracnoideo, posición de la cabeza y desplazamiento en masa del cerebro tras la craneotomía y, sobre todo, por la propia resección tumoral, que incluye fenómenos de expansión generados por la recuperación de espacio perdido por el crecimiento tumoral, edema por manipulación y cambios relativos de presión. La consecuencia es que la reexpansión cerebral hace que la sustancia blanca se vaya acercando al centro de la cavidad generada por la resección tumoral. Este fenómeno de reexpansión cerebral durante la exéresis tumoral ha sido profundamente estudiado por Archip et al. ${ }^{1}$ y Nimsky et al. ${ }^{31,32}$, demostrando con RNM intraoperatoria y TDI con tractografía de la vía corticoespinal que a medida que la cavidad de resección se iba rellenando con parénquima vecino por el fenómeno de reexpansión, los tractos de la vía piramidal se iban aproximando al centro de la misma. La consecuencia de este fenómeno en nuestro protocolo es que se acorta la distancia real entre el punto de EsCD en el lecho de resección tumoral y la situación real del tracto elocuente estimulado, que se ha movido hacia el centro de la cavidad de resección. En ausencia de este sofisticado método de control intraoperatorio de tractografía intraoperatoria propuesto por Archip et al. ${ }^{1} \mathrm{y}$ Nimsky et al. ${ }^{30-32}$, la combinación de los datos proporcionados por la TDI preoperatoria y la EsCD intraoperatoria pueden interpretarse correctamente introduciendo algún factor de corrección para compensar el 'brain shift'. Así, Nimsky et al. ${ }^{31}$ proponen añadir un margen de seguridad al volumen generado por cada tracto que basándose en su propia experiencia calculan entre $5-7 \mathrm{~mm}$.

Conclusiones. El tratamiento de los tumores cerebrales intrínsecos continúa siendo un reto ya que, a pesar del diagnóstico precoz y tratamiento multidisciplinario actual, el crecimiento infiltrativo de dichos tumores conduce indefectiblemente a la recidiva en casi todos los casos con independencia de su tipo o grado. El papel de la cirugía en el tratamiento de estos pacientes se encuentra en constante revisión, pero actualmente se acepta que durante la cirugía se debe maximizar la resección tumoral preservando al máximo las funciones neurológicas relevantes. Los estudios de imagen y funcionales preoperatorios e intraoperatorios permiten desarrollar protocolos específicos de manejo de lesiones en áreas funcionales relevantes integrando los estudios anatómicos y funcionales en los sistemas de neuronavegación. Los grandes retos para estos protocolos son la definición exacta de los límites tumorales y de las áreas funcionales así como el 'brain shift' durante el acto quirúrgico. Nuestro protocolo permite un abordaje seguro a la lesiones intrínsecas del área motora, con resecciones del $90 \%$ del volumen tumoral y con un incremento de los déficits neurológicos transitorios en el postoperatorio inmediato. Está, sin embargo, por definir el impacto que estos protocolos tienen en el periodo libre de enfermedad y supervivencia de los pacientes.

\section{Agradecimientos}

Los autores quieren agradecer la colaboración de todo el personal médico y no médico de los Servicios de Neurocirugía, Neurofisiología y Anestesiología y Reanimación del Hospital Clínico Universitario, Valencia; de ERESA (Exploraciones Radiológicas Especiales), Valencia y del Departamento de Psicología Básica, Clínica y Psicobiología de la UJI, Castellón. Asimismo agradecen el apoyo técnico de MEDTRONIC Ibérica. Este trabajo ha sido financiado en parte por la beca 2008/005 de la Fundación Grupo ERESA para el Desarrollo y la Investigación Médica (Área Neurología).

\section{Bibliografía}

1. Archip, N., Clatz, O., Whalen, S., et al.: Non-rigid alignment of pre-operative MRI, fMRI, and DT-MRI with intraoperative MRI for enhanced visualization and navigation in image-guided neurosurgery. Neuroimage 2007; 35: 609-624.

2. Ávila, C., Barros-Loscertales, A., Parcet-Ibars, M.A., et al.: Aplicaciones de la resonancia nuclear magnética funcional en pacientes prequirúrgicos: funciones motora, de memoria y lingüística. Rev Neurol 2003; 37: 567-578.

3. Berger, M., Deliganis, A., Dobbins, J., Keles, G.E.: The effect of extent of resection on recurrence in patients with low-grade cerebral hemisphere gliomas. Cancer 1994; 15: 1784-1792.

4. Berger, M., Hadjipanayis, C.: Surgery of intrinsic cerebral tumors. Neurosurgery 2007; 61: 279-305.

5. Berman, J., Berger, M., Chung, S.W., Nagarajan, S., Henry, R.G.: Accuracy of diffusion tensor magnetic resonance imaging tractography assessed using intraoperative subcortical stimulation mapping and magnetic source imaging. J Neurosurg 2007; 107: 488-494.

6. Brell, M., Conesa, G., Acebes, J.J.: Estimulación cortical intraoperatoria en el tratamiento quirúrgico de los gliomas de bajo grado situados en áreas elocuentes. Neurocirugía 2003; 14: 491-503.

7. Bürgel, U., Amunts, K., Hoemke, L., Mohlberg, H., Gilsbach, J.M., Zilles, K.: White matter tracts of the human 
brain: Three-dimensional mapping at microscopic resolution, topography and intersubject variability. Neuroimage 2006; 29 : 1092-1105.

8. Claes, A., Idema, A.J., Wesseling, P.: Diffuse glioma growth: a guerrilla war. Acta Neuropathol 2007; 114: 443458.

9. Duffau, H., Gatignol, P., Mandonnet, E., Capelle, L., Taillandier, L.: Intraoperative subcortical stimulation mapping of language pathways in a consecutive series of 115 patients with Grade II glioma in left dominant hemisphere. J Neurosurg 2008; 109: 461-471.

10. Fernández-Miranda, J.C., Rhoton, Jr A,L., AlvarezLinera, J., Kakizawa, Y., Choi, C., de Oliveira, E.P.: Threedimensional microsurgical and tractographic anatomy of the white matter of the human brain. Neurosurgery 2008; 62: 9891028.

11. Fontaine, D., Capelle, L., Duffau, H.: Somatotopy of the supplementary motor area: evidence from correlation of the extent of surgical resection with the clinical patterns of deficit. Neurosurgery 2002; 50: 297-305.

12. Giese. A., Bjerkvig, R., Berens, M.E., Westphal, M.: Cost of migration: invasion of malignant gliomas and implications for treatment. J Clin Oncol 2003; 21: 1624-1636.

13. Gil-Robles, S., Gatignol, P., Lehéricy, S., Duffau, H.: Long-term brain plasticity allowing a multistage surgical approach to World Health Organization Grade II gliomas in eloquent areas. Report of 2 cases. J Neurosurg 2008; 109: 615-624.

14. González-Darder, J.M., González-López, P., Talamantes, F. et al: Multimodal navigation in the functional microsurgical resection of intrinsic brain tumors located in eloquent motor areas: role of tractography. Neurosurg Focus 2010, 28: E5.

15. Harpold. H., Alvord, Jr E., Swanson, K.: The evolution of mathematical modeling of glioma proliferation and invasion. J Neuropathol Exp Neurol 2007; 66: 1-9.

16. Hashiguchi, H., Morioka, T., Yoshida, F., et al.: Usefulness of functional neuronavigation system integrated with functional MRI and corticospinal tractography. Clin Neurophysiology 2007; 118: e189-e202.

17. Joe, B., Fukui, M., Meltzer, C., et al.: Brain tumor volume measurement: comparison of manual and semiautomated methods. Radiology 1999; 212: 811-816.

18. Kamada, K., Sawamura, Y., Takeuchi, F., et al.: Functional identification of the primary motor area by corticospinal tractography. Neurosurgery 2005; [ONS Suppl 1] 56: 98109.

19. Kamada, K., Todo, T., Masutani, Y., Aoki, S., Ino, K., Morita, A., Saito, N.: Visualization of the frontotemporal language fibers by tractography combined with functional magnetic resonance imaging and magnetoencephalography. J Neurosurg 2007; 106: 90-98.

20. Keles, G.E., Chang, E., Lamborn, K., et al.: Volume- tric extent of resection and residual contrast enhancement on initial surgery as predictors of outcome in adult patients with hemispheric anaplastic astrocytoma. J Neurosurg 2006; 105: 34-40.

21. Keles, G.E., Lamborn, K., Berger, M.: Low-grade hemispheric gliomas in adults: a critical review of extent of resection as a factor influencing outcome. J Neurosurg 2001; 95: 735-745.

22. Kim, P., Singh, M.: Functional magnetic resonance imaging for brain mapping in neurosurgery. Neurosurg Focus 2003; 15: 1-7.

23. Kinoshita, M., Yamada, K., Hashimoto, N., et al.: Fiber-tracking does not accurately estimate size of fiber bundle in pathological condition: initial neurosurgical experience using neuronavigation and subcortical white matter stimulation. Neuroimage 2005; 25: 424-429.

24. Maestu, C., Gómez-Utrero, E., Piñeiro, R., Sola, R.G.: Magnetoencefalografía: una nueva técnica de diagnóstico funcional en neurociencia. Rev Neurol 1999; 28: 1077-1090.

25. Mäkelä, J., Forss, N., Jääskeläinen, J., Kirveskari, E., Korvenoja, A., Paetau, R.: Magnetoencephalography in Neurosurgery. Neurosurgery 2006; 59: 493-511.

26. McGirt, M., Chaichana, K., Attenello, F., et al.: Extent of surgical resection is independently associated with survival in patients with hemispheric infiltrating low-grade gliomas. Neurosurgery 2008; 63: 700-708.

27. McGirt, M., Chaichana, K., Gathinji, M., et al.: Independent association of extent of resection with survival in patients with malignant brain astrocytoma. J Neurosurg 2009; 110: $156-162$.

28. Mikuni, N., Okada, T., Enatsu, R., et al.: Clinical impact of integrated functional neuronavigation and subcortical electrical stimulation to preserve motor function during resection of brain tumors. J Neurosurg 2007; 106: 593-598.

29. Mikuni, N., Okada, T., Nishida, N., et al.: Comparison between motor evoked potencial recording and fiber tracking for estimating piramidal tracts near brain tumors. J Neurosurg 2007, 106: 128-133.

30. Nimsky, C., Ganslandt, O., Fahlbusch, R.: Implementation of fiber tract navigation. Neurosurgery 2006; [ONS Suppl 2] 58: 292-304.

31. Nimsky, C., Ganslandt, O., Hastreiter, P., et al.: Preoperative and intraoperative diffusion tensor imaging-based fiber tracking in glioma surgery. Neurosurgery 2005; 56: 130-138.

32. Nimsky, C., Ganslandt, O., Merhof, D., Sorensen, A.G., Fahlbusch, R.: Intraoperative visualization of the piramidal tract by diffusion-tensor-imaging-based fiber tracking. Neuroimage 2006; 30: 1219-1229.

33. Pesudo, J.V., González-Darder, J.M., Feliu, R., Belloch, V., Vera, J., Gil, J.L.: Valoración del grado de resección de los gliomas supratentoriales de alto grado con resonancia magnética postoperatoria precoz. Neurocirugía 2001; 12: 43-50.

34. Price, S.J., Jena, R., Burnet, N.G., et al.: Improved 
delineation of glioma margins and regions of infiltration with the use of diffusion tensor imaging: an image-guided biopsy study. Am J Neuroradiol 2006; 27: 1969-1974.

35. Provenzale, J., McGraw, P., Mhatre, P., Guo, A., Delong, D.: Peritumoral brain regions in gliomas and meningiomas: investigation with isotropic diffusion-weighted MR imaging and diffusion-tensor imaging. Radiology 2004; 232: 451-460.

36. Rhoton, Jr A.L.: The Cerebrum: Anatomy. Neurosurgery $2007 ; 61: 37-118$.

37. Ribas, G.C., Yasuda, A., Ribas, E.C., Nishikuni, K., Rodríguez, A.J. Jr.: Surgical anatomy of microneurosurgical sulcal key points. Neurosurgery 2006; 59 (4 Suppl 2): ONS117-210.

38. Sanai, N., Berger, M.: Glioma extent of resection and its impact on patient outcome. Neurosurgery 2008; 62: 753-766.

39. Sawaya, R., Hammond, M., Schoppa, D., Hess, K.R., Wu, S.Z., Shi, W.M., Wildrick, C.: Neurosurgical outcomes in a modern series of 400 craniotomies for treatment of parenchymal tumors. Neurosurgery $1998 ; 42$ : 1044-1056.

40. Shah, G.D., Kesari, S., Xu, R., et al.: Comparison of linear and volumetric criteria in assessing tumor response in adult high-grade gliomas. Neuro-Oncology 2006; 8: 38-46.

41. Shaw, E.G., Berkey, B., Coons, S., et al.: Recurrence following neurosurgeon-determined gross-total resection of adult supratentorial low-grade glioma: results of a prospective clinical trial. J Neurosurg 2008; 109: 835-841.

42. Stummer, W., Stocker, S., Wagner, S., et al.: Intraoperative detection of malignant gliomas by 5 -aminolevulinic acid-induced porphyrin fluorescence. Neurosurgery 1998; 42: 518-526.
43. Tormos, J.M., Catalá, M.D., Pascual-Leone, A.: Estimulación magnética transcraneal. Rev Neurol 1999; 29: 165-171.

44. Westphal, M., Ram, Z., Riddle, V. et al.: Gliadel wafer in initial surgery for malignant glioma: long-term follow-up of a multicenter controlled trial. Acta Neurochir (Wien). 2006; 148: 269-275.

45. Wu, J., Mao, Y., Zhou, L., et al.: Clinical evaluation and follow-up outcome of diffusion tensor imaging-based functional neuronavigation: a prospective, controlled study in patients with gliomas involving pyramidal tracts. Neurosurgery 2007; 61: 935-949.

46. Yuan, W., Holland, S.K., Jones, B.V., Crone, K., Mangano, F.: Characterization of abnormal diffusion properties of supratentorial brain tumors: a preliminary diffusion tensor imaging study. J Neurosurg Pediatrics 2008; 1: 263-269.

González-Darder, J.M.; González-López, P.; Talamantes-Escribá, F.; García-March, G.; Roldán-Badía, P.; Quilis-Quesada, V.; Verdú-López, F.; Bordes-García, V.; Botella-Maciá, L.; Masbout, G; Cortés-Doñate, V.; Belloch-Ugarte, V.; Ávila, C.: Tratamiento de los tumores cerebrales intrínsecos de áreas motoras elocuentes. Resultados de un protocolo basado en la navegación, tractografía y monitorización neurofisiológica de estructuras corticales y subcorticales. Neurocirugía 2011; 22: 23-35.

Correspondencia: Dr. José M González Darder. Servicio de Neurocirugía. Hospital Clínico Universitario. Avda. Blasco Ibáñez, 17. 46010-Valencia.

gonzalez_jos@gva.es 